\title{
El proceso de incorporación del turismo en la agenda de gobierno de una ciudad
}

\section{The process of incorporating tourism into the government agenda of a city}

ARTÍCULO

\author{
María Carolina Merli \\ Universidad Nacional del Comahue, Argentina. Contacto: mcmerli@gmail.com
}

Recibido: agosto de 2020 Aceptado: septiembre de 2020

\section{Resumen}

El propósito de esta investigación es profundizar el estudio sobre las políticas públicas de turismo implementadas por los gobiernos municipales. Se analiza el caso de la ciudad de Neuquén, Argentina, desde el año 2003 hasta el 2017.

Se caracteriza el proceso de las políticas públicas de turismo de la ciudad de Neuquén en el período mencionado, en base al rol que asume el gobierno municipal, los aspectos que incidieron en la inclusión del turismo en la agenda de gobierno de la ciudad, las formas de incorporación del turismo en la misma y la orientación de las políticas públicas para el desarrollo turístico de la ciudad.

Los conceptos principales que dan un enfoque teórico a este trabajo son las políticas públicas y el turismo como grandes campos teóricos, y la escala de gobierno local como anclaje a los objetivos del estudio.

El proceso de investigación se desarrolla desde un abordaje cualitativo y se adopta un esquema descriptivo. Se analizan diferentes documentos como: ordenanzas, presupuesto municipal, planes y leyes, que se implementaron en el período estudiado.

Finalmente se elaboran conclusiones en relación a las particularidades del caso del estudio y se plantean recomendaciones para la formulación de políticas y la gestión del turismo en la ciudad de Neuquén.

Palabras clave: políticas públicas; turismo; gobierno local; abordaje cualitativo.

\section{Abstract}

The purpose of this research is to deepen the study on public tourism policies implemented by municipal governments. The case of the city of Neuquén, Argentina, from 2003 to 2017 is 
analyzed. The process of public tourism policies of the city of Neuquén in the aforementioned period is characterized, based on the role assumed by the municipal government, the aspects that influenced the inclusion of tourism in the city government agenda, the forms of incorporation of tourism in it and the orientation of public policies for the tourist development of the city.

The main concepts that give a theoretical approach to this work are public policies and tourism as major theoretical fields, and the scale of local government as an anchor to the objectives of the study.

The research process is developed from a qualitative approach and a descriptive scheme is adopted. Different documents are analyzed such as: ordinances, municipal budget, plans and laws, which were implemented in the period studied. Finally, conclusions are drawn up regarding the particularities of the case of the study and recommendations are made for the formulation of policies and the management of tourism in the city of Neuquén.

Keywords: public policies; tourism; local government; qualitative approach.

\section{Introducción}

La ciudad de Neuquén desarrolla un perfil económico volcado a los servicios, dependiente de la actividad hidrocarburífera que se desarrolla en la provincia, las funciones administrativas como ciudad capital, los servicios educativos universitarios, salud de alta complejidad y otras actividades relacionadas como el comercio. El turismo se ha integrado la economía urbana en relación a estas actividades; además de su localización estratégica en relación con las principales vías de acceso a los destinos turísticos de la cordillera.

Es así como ha cobrado relevancia en la agenda de las políticas públicas de la municipalidad. Con algunos antecedentes desde la redacción de la Carta Orgánica Municipal en 1995 y con mayor énfasis a partir del 2004, cuando es posible identificar políticas turísticas concretas. Desde ese entonces, las distintas gestiones de gobierno de la ciudad de Neuquén han considerado el turismo como una actividad socioeconómica de interés para el desarrollo de la ciudad.

El perfil turístico de Neuquén se caracteriza principalmente como centro de estadía (no vacacional) en los meses de marzo a junio y de agosto a diciembre; y centro de escala en los meses vacacionales. En este sentido es posible identificar la presencia de pasajeros en la ciudad, provenientes de otras ciudades del país y de otros países con motivo de desarrollar en Neuquén actividades laborales en empresas relacionadas a la explotación de hidrocarburos. La otra manifestación del turismo que se presenta en la ciudad, son los viajes que tienen por destino final centros turísticos de la cordillera patagónica. Para el caso de los viajes terrestres, la ciudad de Neuquén ha sido un punto estratégico de escala por su localización geográfica y la disponibilidad de servicios para atención de viajeros. Estas dos 
manifestaciones del turismo aún coexisten en la ciudad, junto con otras más recientes. Viajes motivados por la asistencia a congresos y eventos, trámites administrativos, razones de salud y en menor medida, vacaciones.

Esto ha generado expectativas en el sector privado, manifiesto en la creciente ampliación de la capacidad hotelera de la ciudad, la incorporación de circuitos locales y regionales en las agencias de viajes y la habilitación de prestadores de actividades turísticas locales.

En este contexto, el perfil turístico de la ciudad, es vulnerable a la crisis de las otras actividades de las cuales depende. La caída que ha atravesado la actividad petrolera en la región en el período 2014-2016 puesto en evidencia la disminución de los viajes corporativos. A esto se suma la disminución del turismo interno a consecuencia de fluctuaciones macroeconómicas que favorecieron el turismo internacional en ese período. Se observa que si la actividad turística se sostiene a partir de una demanda restringida, ante el sostenido incremento de oferta de plazas hoteleras; se pone en riesgo la rentabilidad de los emprendimientos turísticos de la ciudad, con su consecuente impacto en otros sectores de la economía local.

En este sentido es relevante el estudio de las políticas municipales para el desarrollo del turismo en el ámbito de la ciudad a partir de los siguientes interrogantes:

- ¿Qué rol asume el gobierno local?

- ¿Qué orientación plantean las políticas públicas?

- ¿Cómo se manifiestan en la agenda?

- ¿Por qué se incluyen?

Este estudio se propone interpretar el proceso de las políticas públicas de turismo en la ciudad; en cuanto al rol del gobierno municipal y provincial, los aspectos que incidieron en su inclusión en la agenda de gobierno, las formas de incorporación de políticas turísticas en la agenda municipal y la orientación que de las mismas.

\section{Estado de la cuestión}

En relación a las políticas de los gobiernos municipales orientadas al turismo, hay estudios de referencia en Argentina (Puccio, 2008) y otros países latinoamericanos (Cadena Inostroza y Cruz Jiménez, 2006) y (Giraldo Velásquez y Zapata Aguirre, 2010).

En este sentido, Puccio (2008) considera que los municipios argentinos que tradicionalmente se han desarrollado en la prestación de servicios administrativos y que implementaban políticas surgidas en otros niveles de gobierno, han ganado atribuciones, espacios de poder y autonomía, pero también convergen en ellos intereses políticos de otros 
ámbitos. En ellos, la formulación de política municipales de turismo responde a una mixtura entre un estilo incrementalista, y la intervención de una red de actores que actúa a modo de "filtro selectivo" con las políticas que transmiten los estados provincial y nacional en materia de turismo.

En el caso de los municipios de México considerados en el estudio de Cadena Inostroza y Cruz Jiménez (2006), las dinámicas económicas y sociales también han impulsado a los municipios a participar de manera más activa en la definición de políticas turísticas. Sin embargo, esto pone en evidencia los problemas y limitaciones que caracterizan el ámbito municipal: escasez de recursos, falta de información, insuficiente capacidad administrativa y técnica, falta de independencia política, entre otros.

Por otra parte, en el estudio desarrollado en el departamento de Antioquía, Colombia (Giraldo Velásquez y Zapata Aguirre, 2010), se reconoce el papel fundamental que desempeñan las autoridades regionales y locales en la formulación de políticas y en la organización y desarrollo del turismo para el logro de una mayor competitividad del sector. En este sentido los autores hacen referencia a la importancia de la formulación de un plan de acciones para el sector. Se destaca el rol del gobierno local, aunque el sector privado y la comunidad local también son objeto de atención, por lo que la política turística tiene que ser una acción concertada. El análisis presentado, al igual que el caso de México, pone en evidencia la necesidad de que los municipios sean dotados de mayores recursos y facultades para convertirse en verdaderos gestores estratégicos del potencial turístico.

En estos estudios se reconoce que los gobiernos regionales y locales en países latinoamericanos desempeñan un papel fundamental en la formulación de políticas y en la organización e impulso del turismo. Así como también dan cuenta de su presencia en las agendas de los gobiernos locales y la incidencia de la escala nacional e internacional en esas políticas.

En relación a la articulación del turismo en el territorio, se observaron varios estudios de caso en Argentina, en los cuales la actividad se da como estrategia de complementación económica.

Un caso de referencia en nuestro país es el estudio realizado en Villa Cacique - Barker, provincia de Buenos Aires (Guzmán Ramos y Fernández, 2004), en la cual las políticas públicas se orientan a poner en valor de las instalaciones y tradiciones de la actividad minera como recurso para el desarrollo del turismo. Este estudio considera que los recursos que conforman la historia económica de la ciudad, pueden convertirse en una estrategia alternativa de desarrollo económico local.

Otros estudios a los que puede referirse en nuestro país, son los realizado por Wallingre en la ciudad de Federación, Entre Ríos (2005 y 2010); Ushuaia, Tierra del Fuego (2004); Villa Elisa, Entre Ríos (2011); y Paso de la Patria, Corrientes (2013); ciudades que 
incorporan al turismo en su economía en la intención de complementar o diversificar el perfil económico.

En Villa Elisa, el turismo se sustenta en los recursos termales y la implementación de políticas turísticas fue posible a partir de la transferencia de excedentes de recursos generados por las actividades económicas: agrícola, ganadera, avícola y forestal. En esta localidad, también destaca la formulación de un Plan de Desarrollo Local para el direccionamiento de las políticas económicas de la ciudad. En la localidad de Federación, también se alcanzó la formulación de un Plan de Desarrollo Estratégico Turístico Ambiental como política del gobierno municipal, el cual contribuyó en forma considerable a la diversificación económica sin sustituir a las otras.

Mientras que en Ushuaia, con una marcada impronta de desarrollo industrial y pesquero, la falta de una estrategia de desarrollo de la actividad turística se observó como una dificultad para orientar la diversificación económica. En Paso de la Patria, pueblo que también basaba su economía en la pesca extractiva, el turismo se ha ido fortaleciendo en los últimos años, llegando a cobrar mayor importancia. Sin embargo la localidad tampoco ha logrado formular un lineamiento para el desarrollo de su política turística.

Los estudios mencionados anteriormente dan cuenta de la capacidad del turismo de articularse con otras actividades económicas y con las características de cada territorio; en contextos en los cuales se manifiesta el declive de otras economías. Es por ello que se ha ido convirtiendo en una actividad que despierta interés en los gobiernos municipales y se incluye en la agenda política. Aunque también se observa que cuando su incorporación no es sostenida en planes estratégicos puede ver restringido su alcance e incluso conducir a consecuencias negativas en la comunidad.

También hay antecedentes de estudios de las políticas públicas de la ciudad de Neuquén, en relación al turismo: Suárez (2005), Rodríguez (2011), Bosch y Merli (2011). Estos trabajos analizan la incidencia de las políticas públicas en la transformación del espacio urbano y en las prácticas recreativas.

El estudio de Suárez (2005) pone en evidencia que en el año 2002, el turismo se incluye dentro de la estructura municipal, como un programa social dentro de una subsecretaría y en la denominación de un área de competencia con mayor jerarquía dentro del organigrama. Sin embargo su contenido en cuanto a políticas, no se veía reflejado en las asignaciones presupuestarias.

Por su parte Rodríguez (2011) afirmar que en el proceso de configuración de los espacios turístico - recreativos de la ciudad se ha dado un cambio estético y funcional a partir del gran desarrollo de obras públicas en lugares estratégicos. El estudio considera relevante el análisis de las políticas como una variable significativa para el estudio del espacio urbano. 
Bosch y Merli (2011) ofrecen una caracterización de la ciudad como centro turístico y recreativo, en cuanto a su dinámica vacacional y no vacacional. Además este estudio manifiesta la relevancia de algunos actores que tienen incidencia en las políticas turísticas y destaca la importancia de la articulación de políticas para el desarrollo turístico. Asimismo establece que no se le atribuye marcada relevancia al desarrollo del turismo como actividad socioeconómica de la ciudad, aspecto que se evidencia en la limitación de los recursos asignados.

La cuestión de las políticas de turismo que implementan los gobiernos municipales, parece haber sido abordada con bastante amplitud en nuestro país; y con algunos avances en la ciudad de Neuquén. Este trabajo pretende ahondar en la particularidad que se presenta en las ciudades que sustentan su economía en otras actividades y pretenden instalar el turismo a partir de políticas públicas. Se han mencionado las investigaciones específicas que se pueden encuadrar en esta temática y que se dan en contextos diferentes al de la ciudad que se constituye como caso de estudio.

\section{Abordaje teórico}

Los conceptos ordenadores de este trabajo son las políticas públicas y el turismo como grandes campos teóricos; y la escala de gobierno local como anclaje a los objetivos del estudio. Este planteamiento implica considerar las competencias de los diferentes niveles de gobierno y como se plantea el turismo entre ellas. Además de la incorporación del turismo en la agenda de gobierno.

\section{Políticas públicas}

En una primera aproximación a la noción de políticas públicas se puede afirmar que están orientadas a los ámbitos de la vida colectiva y responden a objetivos establecidos previamente y se materializan a partir de un conjunto de decisiones que se asumen a lo largo de un proceso en el cual se deben utilizan recursos económicos, técnicos, administrativos y normativos para su implementación. Tamayo Sáez, (1997) jerarquiza la figura del gobierno como un conjunto de organizaciones que, utilizan recursos para desarrollar políticas que atienden a los problemas de los ciudadanos, políticas que tienen impactos sociales, políticos y económicos.

Por su parte Subirats define las políticas públicas como un proceso de "decisiones y de acciones resultado de las interacciones estructuradas y repetidas entre diferentes actores, públicos y privados, que por diversas razones están implicados en el surgimiento, 
la formulación y la resolución de un problema políticamente definido como público" ( Subirats, J; Knoepfel, P; Larrue, C y Varonne, F, 2008).

Por otra parte, la producción especializada en políticas turísticas, las define como: "un proceso permanente de toma de decisiones que genera acciones ( $u$ omisiones) que conducen a la definición de un problema y al intento de resolverlo, en donde la autoridad gubernamental es el actor central pero no es el único actor activo, puesto que se mueve en interdependencia con otros" (Villar, 2011)

Los gobiernos son conjuntos de organizaciones de distinta naturaleza y funciones, ejecutivas, legislativas, judiciales, que combinan recursos normativos, humanos, financieros y tecnológicos para la formulación y ejecución de políticas públicas que se representan en programas, servicios públicos, en productos que atienden problemas de los ciudadanos, regulan sus comportamientos y satisfacen sus demandas. En Argentina, según la escala territorial sobre la cual tienen injerencia se identifican organismos de gobierno nacional, provincial y municipal.

Es necesario distinguir las responsabilidades que tiene cada nivel de gobierno respecto de las necesidades de la población que habita en el territorio, éstas son denominadas competencias y se establecen en la Constitución Nacional, las constituciones provinciales y cartas orgánicas municipales. En Argentina están diferenciadas las competencias de los gobiernos municipales en relación con las de los gobiernos provinciales y nacional. Algunas de estas competencias son exclusivas de un nivel de gobierno, es decir que sólo pueden ser ejercidas por el mismo, mientras que otras son concurrentes, es decir que pueden ser adoptadas por uno u otro de acuerdo a la articulación entre los diferentes niveles de gobierno (Cravacuore, 2007). Por otra parte, se distinguen también las funciones de los organismos de gobierno, que no tienen que ver con responsabilidades delegadas o institucionalizadas, sino con aquellas cuestiones en las que el gobierno considera que debe involucrarse o que la población lo asume como tal, lo que el gobierno debe hacer. Éstas se encuentran en el plano de lo simbólico, es subjetivo y se modifican con el tiempo.

El gobierno municipal, que se constituye como la escala de análisis en este estudio, tiene alcance directo en el ejido municipal. Varios autores refieren a gobierno local como sinónimo de gobierno municipal dado que alude a los organismos de gobierno que tienen competencias en el territorio de la menor jurisdicción reconocida constitucionalmente. El gobierno municipal puede ser concebido como un órgano descentralizado del gobierno nacional y provincial con competencias heredadas de estos niveles; aunque también hay líneas teóricas que destacan una posición más autónoma. Por ejemplo Villar (2007), lo concibe como una estructura política del Estado en una sociedad que se autogobierna en el marco de una sociedad mayor. Cabe mencionar que las funciones municipales no están preestablecidas, sino que se van reconstruyendo junto a las expectativas y demandas que generan los cambios sociales y políticos. 
En efecto, Villar (2007) afirma que en el estudio de las políticas municipales de desarrollo económico, entre los cuales se inscribe el turismo, se deben contemplar las políticas implementadas por otros niveles de gobierno. Considera relevante el análisis de la relación que se establece entre las políticas de mayor alcance territorial sobre las de menor alcance. Esto debe considerarse habida cuenta que la agenda política está en permanente construcción y en ese proceso tienen incidencia factores propios del gobierno y la sociedad. Para ello propone analizar el impacto local de las políticas desarrolladas en los otros niveles de gobierno.

\section{El proceso de las políticas públicas}

En el campo de las ciencias políticas se identifican dos tradiciones teóricas diferenciadas que explican el proceso de las políticas públicas, el racionalismo postulada por Lowi (1964) y Allison (1969); y el incrementalismo en la cual se inscribe Lidblom (1959), aunque también hay un tercer enfoque postulado por Amitai Etzioni (1967).

La corriente racionalista asume que es posible confiar en la capacidad humana para desarrollar instrumentos que puedan alterar la realidad y que la política pública deviene de un proceso racional y de planificación. Analiza el problema, en términos técnicos y considera que la solución al problema es producto de la mejor elección, luego de haber evaluado todas las alternativas (Graham, 1992).

El incrementalismo considera que los decisores también están atravesados por valores e intereses y la definición del problema no es netamente técnica. La alternativa de solución se formula en función de los recursos disponibles. En este sentido hay una relación dependiente entre los objetivos y los medios, que se produce en el marco de lo posible. El análisis de las opciones es limitado en función de los recursos disponibles. En el mismo, intervienen múltiples actores e intereses y es posible hacer una caracterización del proceso decisional, la interacción y los intereses que se expresan. Charles E. Lindblom (1959, 1963, 1979) ha sido el principal referente de esta.

Por su parte Amitai Etzioni (1967) plantea un tercer enfoque que impone un estilo balanceado de los dos abordajes. Este autor reconoce que en la política son ineludibles las decisiones fundamentales, estratégicas, del racionalismo, pero considera también que el análisis debe adaptarse a las situaciones específicas y a los recursos disponibles por los decisores. En concordancia con la estrategia racionalista plantea un análisis de fondo de ciertas situaciones y componentes esenciales, pero sin pretender ser exhaustivo en el universo de dimensiones de la cuestión. En esto último se alinea con la estrategia incrementalista, aunque tampoco se reducirá a analizar sólo los componentes que le son conocidos (Aguilar Villanueva, 1992). 
La agenda

Es posible identificar en la literatura abordada, la distinción entre dos tipos de agenda. La agenda sistémica o agenda pública, constituida por el conjunto de problemas que son identificados por la comunidad como situaciones que deberían ser materia de intervención del gobierno (Tamayo Sáez, 1997). Por otro lado, la agenda política, que es el conjunto de problemáticas que son interés de los gobernantes y por lo tanto forman parte de las situaciones a abordar a través de las políticas públicas (Aguilar Villanueva, 1993).

Elder y Cobb (1993) consideran que la formación de la agenda es un proceso muy dinámico en el que interviene un conjunto cambiante de actores, con recursos limitados. Estos autores consideran cuatro aspectos muy dinámicos que inciden en la formación de la agenda: las personas, los asuntos (o problemas), las soluciones y las oportunidades.

\section{Políticas turísticas}

Puede afirmarse que el turismo es práctica social que trasciende las relaciones comerciales y el intercambio económico. Es necesario considerar que la relación que deviene de ésta práctica social involucra múltiples sujetos sociales: los turistas, las comunidades emisoras, las comunidades receptoras, en su extensa diversidad y que actúan según sus intereses (Bertoncello, 2002). En esta dinámica el territorio es el ámbito en el cual se producen los intercambios económicos y sociales, materiales y simbólicos y en el cual se expresan las acciones de los gobiernos locales para favorecer esas relaciones. El Estado se constituye como un actor relevante en esta relación de intercambio porque plantea un marco de condiciones en las cuales se va a desarrollar esa relación. Es por ello que resulta de interés el análisis de las políticas públicas.

Se consideran políticas turísticas municipales a las acciones desarrolladas por los gobiernos de ese nivel para el fomento de la actividad turística en el territorio local, persiguiendo fines de bienestar económico y social del colectivo social que habita en él. También cabe ampliar el alcance de este concepto a las políticas del gobierno municipal que no siendo expresamente turísticas, tienen incidencia en la actividad. Así como también la implementación de políticas de otros niveles de gobierno provincial o nacional en el territorio local. Las políticas públicas, implican un proceso colectivo de construcción y acuerdos en el cual se ven involucrados diferentes agentes de desarrollo de los sectores público y privado involucrados en la actividad turística.

\section{Metodología}


Esta investigación se llevó a cabo desde un enfoque cualitativo; por considerar que las particularidades del objeto de estudio requieren de un abordaje profundo y riqueza interpretativa, en su contextualización específica, para el entendimiento e interpretación de la diversidad de sus manifestaciones posibles (Hernández Sampieri, Fernández-Collado y Baptista , 2006). Se realizó un abordaje descriptivo con la finalidad de aportar características o cualidades del objeto de estudio que son adoptadas en contextos territoriales complejos (Danhke, 1989 en Hernández Sampieri, Fernández-Collado y Baptista, 2006).

El acercamiento al campo de estudio se realiza a partir de la investigación bibliográfica y documental. La técnica utilizada para el trabajo de campo fue el análisis de contenido de documentos oficiales mediante el software Atlas.ti: Carta Orgánica Municipal, ordenanzas relacionadas con el turismo, objetivos y metas anuales, presupuesto general de gastos, normativas provinciales de turismo, planes provinciales de turismo.

\section{Resultados}

Rol del gobierno municipal para el desarrollo turístico de la ciudad

En cuanto a las funciones y competencias del gobierno, el desarrollo turístico se manifiesta como una competencia concurrente entre la escala provincial y municipal. Reconocido en los documentos analizados del siguiente modo:

El texto de la Constitución Provincial (2006) establece como atribución del poder legislativo la reglamentación de la actividad, aunque no se incluye, al menos expresamente, como atribución del poder ejecutivo su desarrollo. "Corresponde al Poder Legislativo: [...] Dictar leyes sobre fomento económico, bosques, turismo, navegación interior, minería, geología y energía hidroeléctrica."

Sin embargo la Ley Provincial de Turismo (2002) da cuenta de que es una función de la autoridad de aplicación (Subsecretaría de Turismo de la Provincia) el desarrollo de la actividad y plantea la vinculación con otras áreas de gobierno, cuyas competencias se relacionen con el turismo. También considera que esas funciones deben ser compartidas con los gobiernos municipales. "A los efectos de desarrollar al turismo como actividad socioeconómica en el ámbito de la Provincia del Neuquén, las autoridades públicas prestarán su apoyo a la autoridad de aplicación de la presente Ley, colaborando en cuanto le corresponda en el ejercicio de sus respectivas competencias vinculadas con el sector [...] La autoridad de aplicación de la presente Ley deberá coordinar con estos municipios acciones respecto de la ejecución de planes, programas y proyectos que coadyuven al desarrollo turístico provincial."

Por su parte, la Carta Orgánica Municipal (1995), establece claramente el desarrollo del turismo como una competencia municipal "Sin perjuicio de las competencias contenidas 
en la Constitución Provincial, esta Carta Orgánica establece las siguientes: [...] Elaborar y ejecutar políticas de turismo".

Sin embargo, hay antecedentes, anteriores a este documento que en el año 1988, plantean la necesidad de crear un área de turismo en la estructura administrativa municipal, asumiendo ya esa función tempranamente. "Facúltase al Departamento Ejecutivo a implementar la Dirección de Turismo Municipal. [...] La Dirección de Turismo Municipal tendrá como misiones fundamentales orientar, fomentar y desarrollar el turismo en el ejido de la Ciudad de Neuquén" (Ordenanza 3890/88)

El análisis de estas categorías pone en evidencia tres aspectos importantes. En primer lugar, que la clara delimitación entre las funciones y competencias, en términos del marco teórico, no se manifiesta tan claramente en ambos niveles de gobierno. En la formulación de la Constitución Provincial, el turismo, se expresa en términos de función, es decir lo que se debe esperar de esa escala de gobierno. Por su parte, el planteamiento de la Carta Orgánica que rige los deberes del gobierno municipal se manifiesta claramente asumiendo al turismo como una competencia; es decir lo que corresponde que sea abordado por las políticas municipales.

En segundo lugar, estas afirmaciones se enriquecen con el antecedente de la Ordenanza citada, que da cuenta de que antes de la formalización del turismo como política de Estado en el gobierno provincial y municipal ya existían antecedentes acerca de la intención de asumir esa función por parte del gobierno local.

\section{Funciones del Estado en materia de Turismo}

La bibliografía especializada (Villar, 2007) refiere a cuatro funciones básicas que desarrolla el Estado en materia de turismo y que se expresan en las políticas públicas: aprovechamiento de recursos del territorio, impulso a la actividad económica, planificación del territorio y regulación de la actividad. Entre las funciones del gobierno municipal se distinguen responsabilidades delegadas del gobierno provincial, como por ejemplo las que tienen que ver con el desarrollo económico y el control de las actividades; así como también cuestiones en las que el gobierno considera que debe involucrarse por sí mismo, por ejemplo el aprovechamiento de los recursos.

Las ordenanzas analizadas al respecto manifiestan una mayor disposición al aprovechamiento de recursos. Esto se explica en consideración del perfil turístico de la ciudad, no dependiente de atractivos turísticos que son el principal recurso del turismo vacacional y que al momento del estudio se encontraban poco desarrollados, sumado a la expectativa de orientar la actividad hacia otros grupos de demanda. Para ello el aprovechamiento de los recursos del territorio implica, entre otras cosas, la puesta en valor de los elementos del patrimonio natural y cultural de la ciudad. Ésta función es la que se 
encuentra más representada en las políticas del gobierno municipal, por hallarse una mayor cantidad de citas en los documentos analizados. La función regulatoria también se manifiesta en las políticas del ejecutivo municipal, aunque no como una función propia de este nivel de gobierno sino como adhesión a normativas provinciales. Por otro lado, el aspecto económico se manifiesta en la fundamentación de una reciente ordenanza en la cual se presenta al turismo como un argumento relevante para el desarrollo de la ciudad, por la oportunidad de diversificación económica respecto del perfil productivo descripto. Finalmente, la función de planificación del territorio se expresa con menor intensidad en las políticas analizadas.

En términos de proceso transcurrido en el período estudiado, se evidencia que la función de aprovechamiento de recursos ha sido desarrollada tempranamente, (años 1997 - 2008); mientras que la regulación de la actividad, el desarrollo económico y la planificación son funciones evidenciadas en periodos más recientes $(2012-2013)$.

\section{El turismo en la agenda de gobierno municipal}

El turismo forma parte de la agenda de políticas públicas de la ciudad de Neuquén, en la formulación de objetivos del órgano de competencia denominado Dirección Municipal de Turismo o Subsecretaría de Turismo en diferentes momentos. También es posible reconocer políticas formuladas por otras áreas del gobierno municipal que guardan relación con la actividad turística; así como políticas formuladas por el órgano legislativo municipal, expresadas en Ordenanzas.

Desde el enfoque cognitivo, cobran relevancia en el análisis de las políticas públicas las ideas y representaciones de los gobernantes en la interpretación de los problemas, considerando que a partir de la interpretación del problema en cuestión se formulan los objetivos en base a los cuales se orienta la política. El análisis desde este marco le da un especial valor a la argumentación y los valores que se reflejan en la política pública. ( Subirats, J; Knoepfel, P; Larrue, C y Varonne, F, 2008)

Considerando que cada organismo tiene sus competencias, funciones y valores, la formulación de políticas turísticas jerarquiza diferentes cuestiones que se muestran a continuación. 
Gráfico 1: Contenido de las políticas turísticas municipales representado en ordenanzas municipales según cantidad de menciones. Ciudad de Neuquén, 2003 - 2017.

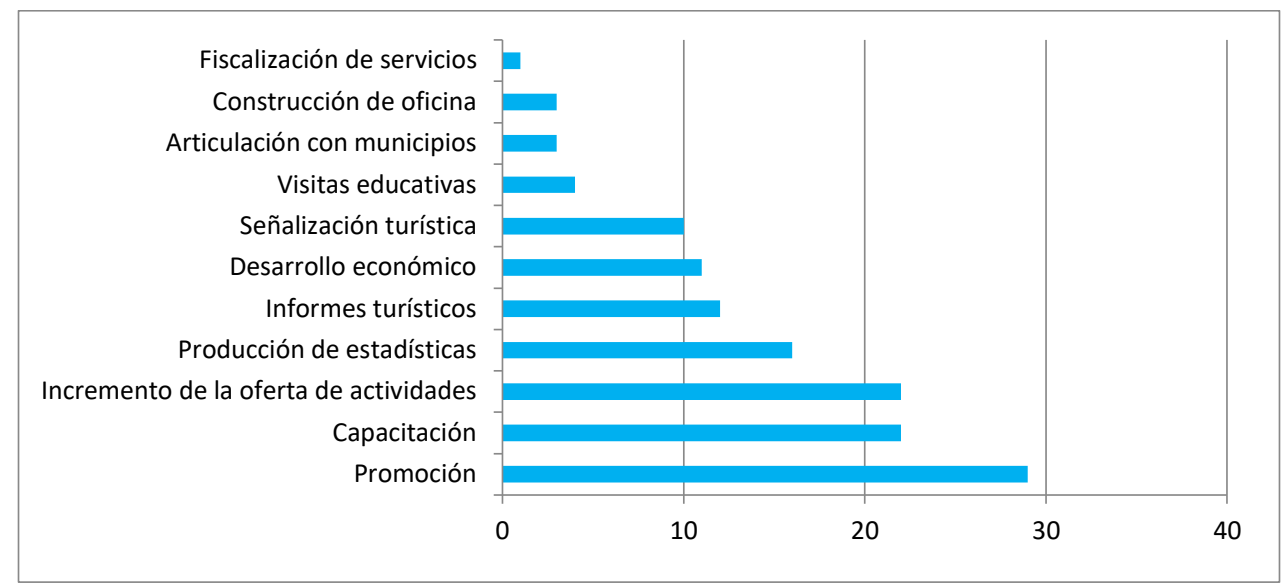

Fuente: (Merli, 2018) "El proceso de incorporación del turismo en la agenda de gobierno de una ciudad. Estudio de caso: Ciudad de Neuquén". Tesis de maestría Ciencias Sociales y Humanidades. Universidad Nacional de Quilmes.

En los objetivos formulados por la Subsecretaría de Turismo municipal las políticas turísticas que manifiestan mayor contenido son las de promoción y capacitación.

Como se mencionó anteriormente, el turismo también es considerado en la formulación de objetivos anuales de otras áreas del gobierno local. Estas áreas, si bien se orientan a la atención de otras necesidades de la vida urbana, formulan políticas de diferente naturaleza que pueden incidir en la actividad turística. Como se mencionó en el marco teórico, pueden distinguirse las políticas turísticas de las políticas relacionadas al turismo (Villar, 2011). Éstas últimas son originadas por otros organismos de gobierno y de algún modo inciden en alguno de los elementos del sistema turístico. En el caso particular de estudio se considera relevante considerarlas porque ponen en evidencia que el turismo se presenta y se valora como una actividad socioeconómica de la ciudad, aún en áreas de gobierno que están abocadas a la atención de necesidades propias de la población. Esto se evidencia en la formulación de objetivos anuales de otras dependencias de gobierno municipal como la Subsecretaría de Deportes, la Subsecretaría de Acción Social, Dirección de Atención al Ciudadano, Subsecretaría de Cultura, Estación Terminal de Ómnibus Neuquén, Museo Nacional de Bellas Artes Neuquén, Subsecretaría de coordinación, Dirección Municipal de Protocolo, Subsecretaría de Relaciones Internacionales y Defensa Civil.

Aspectos que inciden en la formación de la agenda

La revisión bibliográfica da cuenta de que la agenda de gobierno es muy dinámica y se construye a partir de una amplia variedad de factores del contexto inmediato y de otros contextos más amplios que inciden en los intereses y preocupaciones de los gobernantes. En los documentos analizados se identificaron algunas categorías de las planteadas por los autores (Lowi, 1992; Elder y Cobb, 1993): la incidencia de asuntos de la agenda sistémica, 
la influencia de grupos de poder, la influencia de otras agendas, las oportunidades, los recursos disponibles, las soluciones preexistentes y los valores de los gobernantes.

Gráfico 2: Aspectos que incidieron en la inclusión del turismo en la agenda de gobierno municipal. Ciudad de Neuquén, $2003-2017$.

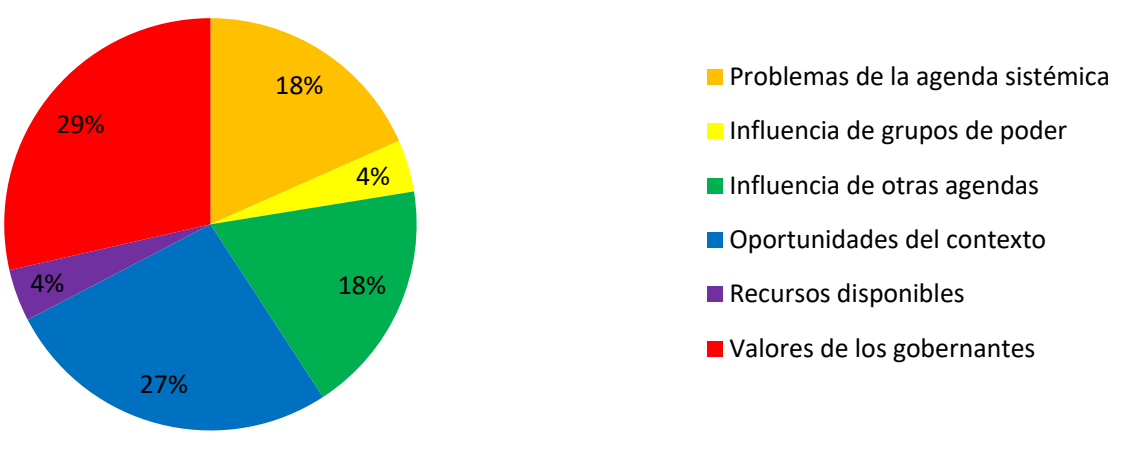

Fuente: (Merli, 2018) "El proceso de incorporación del turismo en la agenda de gobierno de una ciudad. Estudio de caso: Ciudad de Neuquén". Tesis de maestría Ciencias Sociales y Humanidades. Universidad Nacional de Quilmes.

Los aspectos que se manifiestan en su mayoría refieren a valores de los gobernantes, en este caso los legisladores, que se expresan en los considerandos de las ordenanzas formuladas. En segundo lugar la presencia de oportunidades del contexto que resultan favorables para la inclusión del turismo en la agenda de gobierno y en tercer lugar los asuntos o problemas de la agenda sistémica y la influencia de otras agendas. Como se mostró en el gráfico existen otros aspectos que inciden en la formación de la agenda, aunque en el caso de estudio se mostraron con menor relevancia: soluciones preexistentes, recursos disponibles y grupos de poder.

Como postulan los autores trabajados (Lowi, 1992), en el proceso de formación de la agenda de gobierno, la decisión de incluir un problema en ella expresa la posición ideológica, la formación profesional, entre otros aspectos de los gobernantes. Estas valoraciones se expresan más acentuadamente cuando es limitado el conocimiento del problema. En la fundamentación de las ordenanzas analizadas es posible identificar los valores de los gobernantes que se expresan en la formulación de las políticas. Cabe destacar el reconocimiento del turismo como derecho, la importancia atribuida al patrimonio histórico y cultural, el rol importante que cumplen las personas que están en contacto directo con los turistas, las ventajas que representa el turismo para la ciudad, la valoración de obras públicas que favorecen el desarrollo turística, la importancia de adoptar criterios de sustentabilidad en el desarrollo económico, entre otros.

En cuanto a las oportunidades del contexto que se presenta como el segundo factor de influencia representado en las Ordenanzas, los autores referidos (Elder, Ch. y Cobb, R., 1993) mencionan que en la agenda suelen presentarse momentos de mayor receptividad atribuidos a diferentes razones como por ejemplo: momentos electorales o el primer año de gestión. A estas oportunidades se les denominan ventanas políticas. En el caso en estudio, 
fue posible identificar situaciones del contexto más inmediato que favorecieron la instalación de temas en la agenda. Algunas cuestiones se vinculan con la dinámica propia de la ciudad, como por ejemplo: la inversión en infraestructura hotelera, así como obras públicas que favorecen la puesta en valor de los atractivos turísticos. También se identifican como oportunidades aspectos en los cuales la ciudad presenta ventajas en el contexto nacional, como el posicionamiento turístico que tiene el país y la región Patagonia para el turismo internacional. Finalmente se reconoce a los actuales grupos de pasajeros que arriban a nuestra ciudad por diferentes razones, como una oportunidad para fortalecer el desarrollo turístico.

Como formulan los autores, la agenda sistémica o agenda pública, es el conjunto de asuntos o problemas que se presentan como una preocupación para la comunidad y los actores del gobierno prestan intervención cuando diferentes factores como los grupos de poder, los medios, otras agendas inciden como sesgos positivos (Lowi, 1992). Estos aspectos se evidencian en la fundamentación de las ordenanzas municipales, en la mención de asuntos o problemas de la sociedad que se constituyen en el objeto de políticas públicas. Entre ellos se destacan lugares con atractivo natural que no son conocidos por los turistas, necesidad de prolongar la estadía del pasajero, los impactos negativos que produce el turismo en el ambiente, existencia de establecimientos turísticos no habilitados, la necesidad de diversificar la economía local, etc.

La influencia de otras políticas también se expresa como un factor de construcción de la agenda de la ciudad. El autor de referencia (Lowi, 1992) evidencia que existen predisposiciones para tratar temas que forman parte de agendas federales, con modificaciones incrementales en las escalas menores de gobierno. Sin embargo, en los documentos analizados, también se hace referencia a políticas de organismos internacionales como la Organización de las Naciones Unidas o la Organización Mundial del Turismo y el Consejo Internacional sobre Monumentos y Sitios, manifestando su adhesión a las mismas en la escala de la ciudad de Neuquén. También se expresan lineamientos de políticas nacionales que generan incentivo para políticas del ámbito local. Asimismo se identifican políticas provinciales propias de las funciones y competencias de ése nivel de gobierno, que incentivan voluntad de adhesión en la ciudad. Estas se refieren fundamentalmente a la regulación de los servicios turísticos.

Relación entre las políticas turísticas municipales y las provinciales

Considerando lo analizado anteriormente en cuanto a la incidencia de otras agendas en la instalación de temas, se profundizó este aspecto a través del contenido de las políticas turísticas que proponen las agendas de la gestión del ejecutivo local, el órgano legislativo municipal y el ejecutivo provincial. En términos teóricos, esta relación pone en evidencia la 
articulación de las políticas turísticas en los diferentes organismos que forman parte del subsistema de gestión del turismo (Bosch, J. L. y Merli, M. C. , 2014); generando mejores perspectivas para el desarrollo de la actividad, habida cuenta de que las políticas alcanzan mayor nivel de consenso y mejores posibilidades de concreción ante la mejor disponibilidad de recursos. Los datos presentados manifiestan que los contenidos que guardan correspondencia en las tres agendas consideradas son los vinculados a la promoción, capacitación, desarrollo de la oferta y señalización turística.

Se puede afirmar que ha existido una muy baja relación entre las políticas turísticas municipales y los lineamientos provinciales. Existen unos pocos temas en común en ambas agendas, como la necesidad de ampliación de actividades turísticas, entre las cuales se individualiza el turismo cultural y el turismo de reuniones, además de la señalización de lugares turísticos. La mayor cantidad de relaciones se hallaron en relación con el Plan de Desarrollo Turístico Provincial 2008-2011.

También se evidencia una débil relación entre los contenidos de las políticas de turismo del ejecutivo local y el contenido de las ordenanzas municipales (legislativo), dado que sólo se presentaron dos temas en común en ambas agendas. Se identifican dos normativas que tienen su correlato en los objetivos anuales, por un lado la Ordenanza $N^{\circ} 12811 / 13$ que atribuye la facultad al órgano ejecutivo para la creación de un Ente Mixto de Promoción Turística y la Ordenanza $N^{\circ} 11040 / 08$ que propone la creación del Programa de Capacitación para taxistas y auxiliares de taxis.

Orientación de las políticas públicas para el desarrollo turístico de la ciudad

Se analizaron las políticas turísticas en base a la orientación que plantean sus objetivos (Villar, 2007), tomando las categorías: orientación a la calidad, orientación a la competitividad, orientación a la gestión, orientación a la promoción y orientación a la sustentabilidad.

Gráfico 3: Orientación de las políticas para el desarrollo turístico (porcentaje). Ciudad de Neuquén 2003 - 2017.

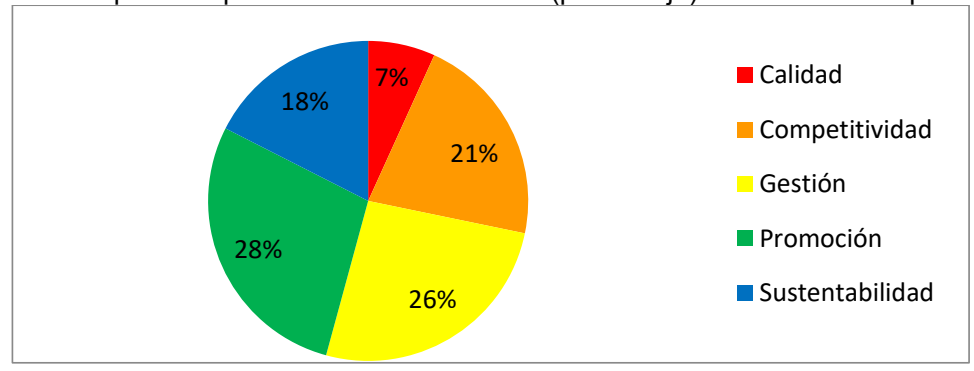

Fuente: (Merli, 2018) "El proceso de incorporación del turismo en la agenda de gobierno de una ciudad. Estudio de caso: Ciudad de Neuquén”. Tesis de maestría Ciencias Sociales y Humanidades. Universidad Nacional de Quilmes.

El contenido de los documentos analizados pone en evidencia que las políticas públicas de la escala municipal, para el desarrollo turístico de la ciudad se orientan 
principalmente a la promoción turística, en segundo lugar a la gestión, luego a la competitividad, la sustentabilidad y finalmente la calidad.

Las políticas de promoción que en términos teóricos tienen por finalidad difundir en la demanda real y potencial, la oferta del centro turístico, se expresan en el caso de estudio con diferentes estrategias. Las mismas comienzan a manifestarse en el año 2008 y presentan continuidad en diferentes períodos de gestión, como así lo manifiestan los objetivos anuales. Se caracterizan por realizar difusión de actividades y servicios turísticos en eventos específicos y de otra naturaleza que se realizan en la ciudad, la región y otras ciudades del país, además de realizar giras de promoción en países limítrofes y la creación de un organismo mixto específicamente abocado a la promoción turística. También se manifiestan con bastante políticas orientadas a la gestión, con el objetivo de lograr la disposición de recursos y estructuras para el desarrollo de la actividad. En el análisis de los documentos considerados, esta categoría se representa en acciones direccionadas a capacitación del personal, disposición de oficinas propias, fortalecimiento institucional, producción de información para la toma de decisiones, servicio de informes turísticos y lograr la representatividad del área de competencia en instancias asociativas de temáticas turísticas. La orientación a la competitividad se evidencia en acciones vinculadas al el desarrollo de servicios turísticos, de productos, de actividades y atractivos y el posicionamiento del destino. Finalmente, la orientación a la sustentabilidad, que refleja el resguardo de los impactos del turismo en diferentes dimensiones, en el caso de estudio se evidencia en políticas de desarrollo económico, de turismo social y la puesta en valor de atractivos turísticos.

\section{Conclusiones}

Este trabajo expone el proceso de incorporación y trayectoria de las políticas públicas de turismo en el gobierno municipal de la ciudad de Neuquén. Para ello analiza cual ha sido el rol del estado municipal y provincial en el desarrollo turístico de la ciudad, así como la orientación que han asumido las políticas públicas. También se desarrollan los aspectos que incidieron en la formación de la agenda de políticas turísticas y la presencia que han tenido en el presupuesto y el organigrama municipal.

Del análisis de las funciones y competencias del gobierno, se deduce que el turismo es una competencia concurrente de la escala provincial y municipal. Se expresa como tal en la Carta Orgánica Municipal, y se manifiesta como una función legislativa en la Constitución Provincial. Cabe considerar los conceptos de Wallingre (2014) quien manifiesta que los gobiernos locales presentan ventajas, para elaborar sus proyectos de desarrollo turístico, en el contexto del fortalecimiento de los municipios a partir del espacio cedido en otros ámbitos institucionales. En la ciudad de Neuquén, el turismo se ha manifestado en la agenda política 
como una actividad de interés para la ciudad desde fines de la década del 80' y ha cobrado un interés creciente. Esta particularidad del caso de estudio se explica en el actual contexto político institucional de nuestro país, en el cual las funciones municipales no están preestablecidas. Por el contrario, se encuentran determinadas por cambios sociales, políticos e institucionales del contexto inmediato y de contextos nacionales, que reconstruyen las expectativas de las comunidades. Al respecto, la crisis económica y la inflación como condiciones del contexto económico de nuestro país, han desalentado el turismo interno como producto del elevado precio de los servicios turísticos en relación a los de algunos destinos internacionales, favoreciendo además, los viajes al extranjero. Aunque también la relación cambiaria con el dólar ha favorecido el ingreso de turistas provenientes de otros países. En el contexto más regional, cabe destacar que la crisis económica tuvo principal impacto en la actividad petrolera, disminuyendo su aporte a la economía local. Por tratarse de una de las principales actividades del sector privado que dinamiza el comercio y los servicios. Esto ha llevado al replanteamiento de las estructuras económicas y la necesidad de diversificación. Para lo cual el turismo genera expectativas favorables.

En cuanto a las funciones del Estado en materia de políticas turísticas, el análisis formulado en base a las categorías de Villar (2007), pone en evidencia que existe una acentuada distinción del aprovechamiento de recursos, como la función más representada por el organismo de turismo del gobierno municipal. El área de competencia ha manifestado su preocupación por la utilización de los recursos turísticos del territorio. En este sentido se consideraron los elementos del patrimonio natural y cultural, así como inversiones públicas o privadas que ponen en valor los atractivos turísticos. También se evidencian otras funciones, como la regulación de la actividad o la planificación del territorio, aunque con menor protagonismo. Las experiencias de otros municipios como Villa Cacique - Barker, provincia de Buenos Aires, (Guzmán Ramos, A.; Fernández, G., 2004) manifiestan asimismo, que la función más desarrollada por el gobierno municipal fue la puesta en valor de los recursos del territorio, básicamente elementos del patrimonio cultural, aunque en ese caso asociados a la actividad minera tradicional.

En función de la problemática planteada, se evidencia que en el caso de estudio, el ejercicio del poder está expresado en políticas de incentivo. Esto se explica en la búsqueda de acrecentar el alcance económico del turismo como una actividad complementaria a la matriz local. En este caso el Estado, representado en el gobierno local, como actor principal de este proceso, intenta promover la actividad turística en el ámbito de la ciudad incentivando a diferentes actores que forman parte del sistema turístico, como sectores gubernamentales no vinculados al turismo en forma directa, asociaciones empresarias, colegio de profesionales, prestadores de servicios turísticos, por mencionar algunos. Al respecto, refiere Puccio (2008) que los municipios argentinos han generado autonomía y distribuciones respecto de su rol tradicional de prestadores de servicios urbanos e 
implementación de políticas de otros niveles. Aunque también se presentan como ámbitos de convergencia de intereses político de otros niveles, en el que coexisten un estilo incrementalista en la elaboración de políticas públicas, una red de actores públicos y privados, que en virtud de sus intereses sectoriales y afinidades actúa como filtro de políticas de otros niveles de gobierno.

Respecto de la orientación en relación a los objetivos para el desarrollo turístico, en función de las categorías planteadas por Villar (2007), en el período estudiado se manifiesta una mayor cantidad de políticas orientadas a la promoción y en segundo lugar a la gestión. Estas se expresan en los objetivos de gestión formulados para el período 2004 - 2017; mientras que las ordenanzas se orientan con mayor énfasis a la sustentabilidad. Esta diferenciación, en parte, se explica en la composición institucional y las funciones propias de cada organismo de gobierno. Mientras que las ordenanzas son elaboradas por una pluralidad de actores que representan diferentes fuerzas de poder que forman parte de la arena política, los objetivos anuales se desarrollan directamente desde el área de competencia del ejecutivo local como actor principal. Este último escenario presenta menor conflictividad al momento de delinear políticas públicas. También es notable la referencia al turismo en políticas de otras dependencias del gobierno local orientadas a la prestación de beneficios directos a la población como cultura o deportes, así como áreas que se destinan a la relación con otras dependencias, reflejando las expectativas y valores de los gobernantes en cuanto al turismo.

Considerando los aspectos que inciden en la formación de la agenda que proponen los autores Tamayo Sáez (1997) y Lowi (1992), en el contenido de las ordenanzas municipales se evidencia que los valores de los gobernantes y las oportunidades del contexto son los aspectos más reflejados. Cabe mencionar que esta categoría no ha sido estudiada en las investigaciones de referencia en materia de políticas turísticas y resulta de gran riqueza su análisis.

Además se realiza un análisis pormenorizado respecto del contenido de las políticas turísticas para evaluar la relación entre las emanadas desde el órgano ejecutivo y legislativo local; y el ejecutivo provincial. Los contenidos en relación con la promoción turística son los más representados en la agenda del ejecutivo local, en consonancia con la orientación predominante. Mientras que en las otras agendas mencionadas los contenidos más desarrollados son los relativos al desarrollo de la oferta turística. Cabe reiterar que las ordenanzas plantean políticas de puesta en valor de atractivos, aspecto que constituye la oferta; así como también la planificación provincial desarrolla el espectro de productos turísticos para todo el territorio provincial, dentro del cual se especifican los propios de la ciudad de Neuquén.

También es posible identificar en este estudio las relaciones de correspondencia entre los contenidos de las políticas municipales, según se originen en el órgano ejecutivo o el 
legislativo y las políticas provinciales. Se considera que el grado de relación es relativamente bajo dado que se circunscribe a unos pocos temas de la agenda y se formulan en períodos anacrónicos. Estos vínculos se explican en la influencia que ejercen las agendas de unos organismos en otros para la instalación de temas, considerando la competencia que existe entre diferentes actores para el tratamiento de asuntos que despiertan particular interés por parte de la población o las oportunidades que se presentan como ventanas políticas. Estas ideas afirman el planteamiento de Puccio (2008) y Rodríguez (2011) en relación con la complejidad de los territorios urbanos en cuanto a la diversidad de actores e intereses que convergen y en relación con la construcción del espacio urbano de la ciudad de Neuquén desde las políticas y la gestión.

En cuanto al proceso de las políticas públicas puede afirmarse que en la agenda del ejecutivo local se presentan continuidades dentro del período estudiado. Esto se explica en actividades propias de la gestión básica como la atención de oficinas de informes, las estadísticas de turismo y la promoción. La formulación de estas políticas se explica desde el modelo incrementalista, que considera las limitaciones institucionales como barreras para formular soluciones innovadoras, y consecuentemente se adopta la estrategia de seguir haciendo lo que está probado que funciona. Esta idea reafirma la hipótesis de Puccio (2008) en relación con los municipios argentinos, al considerar, que el estilo incrementalista convive con políticas turísticas emanadas de los estados provincial y nacional.

En relación con la problemática planteada puede afirmarse que el turismo está claramente representado en la agenda de gobierno del ejecutivo local, como así también en las ordenanzas municipales, aunque con orientaciones diferentes. La actividad ya integraba la economía urbana desde períodos anteriores al estudio, aunque no era objeto de políticas públicas. La consideración surge como consecuencia de la crisis de otras actividades económicas y condiciones favorables del contexto nacional e internacional, sumado a procesos institucionales del país, de la política turística provincial, expresada en la planificación y de condiciones propias del gobierno local. En cuanto a esto último, cabe mencionar la profesionalización del área con la inserción de profesionales y el reconocimiento político alcanzado con la jerarquización del área.

\section{Referencias bibliográficas}

Aguilar Villanueva, L. (1992). Estudio introductorio. En L. Aguilar Villanueva, T. Lowi, G. Allison, C. Lindblom, D. Yehezkel, E. Amitai, A. Meltzner. La hechura de las políticas públicas. México D.F.: Miguel Angel Porrúa.

Aguilar Villanueva, L. (1993). Problemas públicos y agenda de gobierno. México D. F.: Porrúa.

Bertoncello, R. (2002). Turismo y territorio. Otras prácticas, otras miradas. Aportes y Transferencias 6, 29-50. 
Bosch, J. L. y Merli, M. C. (2014). La articulación en el proceso de las políticas públicas del turismo. Realidad, Tendencias y Desafíos en Turismo CONDET 12, 59-73.

Bosch, J. L., \& Merli, M. C. (2011). Estudio de las políticas públicas para el turismo y la recreación en la ciudad de Neuquén. Neuquén: Educo.

Cadena Inostroza, C., \& Cruz Jiménez, G. (2006). Políticas públicas municipales, relación de actores y desarrollo turístico en dos localidades del estado de México. Toluca: El Colegio Mexiquense.

Congreso General Constituyente. (1994). Infoleg. Recuperado de http://servicios.infoleg.gob.ar/infolegInternet/anexos/0-4999/804/norma.htm

Convención General Constituyente. (2006). Sistema Argentino de Información Jurídica. Recuperado de http://www.saij.gob.ar/legislacion/constitucion-neuquen-2006.htm

Convención Municipal Constituyente. (1995). Concejo Deliberante de la Ciudad de Neuquén. Recuperado el mayo de 2016, de http://www.cdnqn.gov.ar/wpcontent/uploads/linksutiles/carta-organica-neuquen.pdf

Cravacuore, D. (2007). Los municipios argentinos (1990 - 2005). En D. Cravacuore, \& R. Israel. Procesos políticos comparados en municipios de Argentina y Chile (19902005). Bernal: Universidad Nacional de Quilmes.

Elder, Ch. y Cobb, R. (1993). Formación de la agenda. En L. y. Aguilar Villanueva. Problemas públicos y agenda de gobierno (pp. 77-104). México D.F.: Miguel Ángel Porrua.

Giraldo Velásquez, C. M., \& Zapata Aguirre, S. (2010). Política turística del departamento de Antioquía. Revista TURyDES 3 (8).

Gobierno de la Provincia de Neuquén. (2004). Neuquentur. Recuperado de http://neuquentur.gob.ar/es/leyes-y-reglamentaciones/

Guzmán Ramos, A.; Fernández, G. (2004). El patrimonio industrial-minero como recurso turístico cultural: El caso de un pueblo-fábrica en Argentina. PASOS Revista de Turismo y Patrimonio Cultural 2, 101 - 109.

Hernández Sampieri, Fernández-Collado y Baptista (2006). Metodología de la Investigación $4^{a}$ Edición. México: Mc Graw-Hill.

Legislatura de la Provincia de Neuquén. (2004). Neuquentur. Recuperado de http://neuquentur.gob.ar/es/leyes-y-reglamentaciones/

Legislatura de la Provincia del Neuquén. (2002). Ley N²414. Ley Provincial de Turismo. Neuquén, Argentina.

Lowi, T. (1992). Políticas Públicas, estudio de caso. En L. Aguilar Villanueva, T. Lowi, G. Allison, C. Lindblom, D. Yehezkel, E. Amitai, A. Meltzner. La hechura de las políticas públicas (pp. 89-117). Distrito Federal: Porrua.

Ministerio de Turismo. Gobierno de la Nación. (2016). Turismo.gov.ar. Recuperado de http://www.turismo.gov.ar/sites/default/files/plan_integral_de_gestion_2016-19vf.pdf

Puccio, H. (2008). La gestión del turismo en municipios turísticos. El diseño de la agenda de cuestiones. IX Jornadas Nacionales y III Simposio Internacional de Investigación - Acción en Turismo. San Juan.

Rodríguez, M. D. (2011). Pensar y Hacer la Ciudad. Neuquén: Educo. 
Subirats, J; Knoepfel, P; Larrue, C y Varonne, F. (2008). Análisis y gestión de políticas públicas. Barcelona: Ariel.

Suárez, S. (2005). Políticas del ocio a escala municipal ¿democracia cultural?. Estudio de caso ciudad de Neuquén. Neuquén: Tesis de maestría en Teorías y Políticas de la Recreación. Facultad de Turismo. Universidad Nacional del Comahue.

Subsecretaría de Turismo Municipalidad de Neuquén (2014). Anuario Estadístico de Turismo Ciudad de Neuquén 2013. Neuquén.

Subsecretaría de Turismo Municipalidad de Neuquén (2015). Anuario Estadístico de Turismo Ciudad de Neuquén 2014. Neuquén.

Subsecretaría de Turismo Municipalidad de Neuquén (2016). Anuario Estadístico de Turismo Ciudad de Neuquén 2015. Neuquén.

Subsecretaría de Turismo Municipalidad de Neuquén (2017). Anuario Estadístico de Turismo Ciudad de Neuquén 2016. Neuquén.

Subsecretaría de Turismo Municipalidad de Neuquén (2017). Informe estadístico de ocupación hotelera $1^{\circ}$ trimestre 2017. Neuquén.

Subsecretaría de Turismo Municipalidad de Neuquén (2017). Informe estadístico de ocupación hotelera $2^{\circ}$ trimestre 2017 . Neuquén.

Subsecretaría de Turismo. Ministerio de Desarrollo Territorial (2011). Plan de Desarrollo Turístico Provincial 2011 - 2015. Neuquén.

Subsecretaría de Turismo. Ministerio de Desarrollo Territorial (2008). Plan de Gestión de Turismo 2008 - 2011. Neuquén, Argentina.

Subsecretaría de Turismo. Ministerio de Producción y Turismo (2003). Plan Maestro de Turismo 2003 - 2007. Neuquén, Argentina.

Subsecretaría de Turismo. Municipalidad de Neuquén (2017). Informe estadístico de ocupación hotelera $3^{\circ}$ trimestre 2017. Neuquén.

Subsecretaría del Turismo Municipalidad de Neuquén (2013). Anuario Estadístico de Turismo 2012. Neuquén.

Tamayo Sáez, M. (1997). El análisis de las políticas públicas. En R. Bañon, \& E. Carrillo, La nueva administración pública. Madrid: Alianza Universidad.

Villar, A. (2007). Políticas municipales para el desarrollo económico - social: revisando el desarrollo local. Buenos Aires: CICCUS.

Villar, A. (2011). Políticas públicas en turismo. En N. Wallingre, \& A. Villar, Desarrollo y gestión de destinos turísticos. Políticas y estrategias (pp. 51-74). Bernal: Universidad Nacional de Quilmes.

Wallingre, N. (2004). El turismo como factor de reconversión productiva local en la ciudad de Ushuaia, República Argentina. Anuario Turismo y Sociedad V(3), 86-94.

Wallingre, N. (2005). El turismo como factor de reconversión productiva local en la ciudad de Federación, Argentina. Revista Aportes y Transferencias. Centro de Investigaciones Turísticas 9(1), 35-55.

Wallingre, N. (2011). El desarrollo de un nuevo destino turístico. El caso de la ciudad de Villa Elisa, Argentina. Anuario Turismo y Sociedad VII, 235-259. 
Wallingre, N. (2013). Análisis del desarrollo de un destino turístico emergente: el caso de la ciudad de Paso de la Patria, provincia de Corrientes, Argentina. Tiempo de Gestión 16, 9-36.

Wallingre, N. (2014). Definición y parámetros de identificación de municipios turísticos. En N. Wallingre, \& A. Villar, Gestión de Municipios Turísticos (pp. 17-39). Bernal: Universidad Nacional de Quilmes.

Wallingre, N. (2014). Instrumentos para el diagnóstico integral de la oferta en destinos turístico. En N. Wallingre, \& A. Villar, Gestión de Municipios Turísticos.

Instrumentos 